Topological Methods in Nonlinear Analysis

Volume 46, No. 2, 2015, 617-630

DOI: $10.12775 /$ TMNA.2015.061

(C) 2015 Juliusz Schauder Centre for Nonlinear Studies

Nicolaus Copernicus University

\title{
EXISTENCE OF SOLUTIONS FOR A KIRCHHOFF TYPE \\ FRACTIONAL DIFFERENTIAL EQUATIONS \\ VIA MINIMAL PRINCIPLE AND MORSE THEORY
}

\author{
Nemat Nyamoradi - Yong Zhou
}

AbSTRACT. In this paper by using the minimal principle and Morse theory, we prove the existence of solutions to the following Kirchhoff type fractional differential equation:

$$
\left\{\begin{aligned}
& M\left(\int_{\mathbb{R}}\left(\left|-\infty D_{t}^{\alpha} u(t)\right|^{2}+b(t)|u(t)|^{2}\right) d t\right) \\
& \cdot\left({ }_{t} D_{\infty}^{\alpha}\left(-\infty D_{t}^{\alpha} u(t)\right)+b(t) u(t)\right)=f(t, u(t)), \quad t \in \mathbb{R}, \\
u \in & H^{\alpha}(\mathbb{R}),
\end{aligned}\right.
$$

where $\alpha \in(1 / 2,1),{ }_{t} D_{\infty}^{\alpha}$ and ${ }_{-\infty} D_{t}^{\alpha}$ are the right and left inverse operators of the corresponding Liouville-Weyl fractional integrals of order $\alpha$ respectively, $H^{\alpha}$ is the classical fractional Sobolev Space, $u \in \mathbb{R}, b: \mathbb{R} \rightarrow \mathbb{R}$, $\inf _{t \in \mathbb{R}} b(t)>0, f: \mathbb{R} \times \mathbb{R} \rightarrow \mathbb{R}$ Carathéodory function and $M: \mathbb{R}^{+} \rightarrow \mathbb{R}^{+}$is a function that satisfy some suitable conditions.

2010 Mathematics Subject Classification. 34C37, 35A15, 35B38.

Key words and phrases. Fractional differential equations, minimal principle, Morse theory, solutions, Critical point theory. 


\section{Introduction}

The aim of this paper is to establish the existence of nontrivial solutions for the following Kirchhoff type fractional differential problem

$$
\left\{\begin{array}{l}
M\left(\int_{\mathbb{R}}\left(\left|-\infty D_{t}^{\alpha} u(t)\right|^{2}+b(t)|u(t)|^{2}\right) d t\right) \\
\quad \cdot\left({ }_{t} D_{\infty}^{\alpha}\left(-\infty D_{t}^{\alpha} u(t)\right)+b(t) u(t)\right)=f(t, u(t)), \quad t \in \mathbb{R}, \\
u \in H^{\alpha}(\mathbb{R}),
\end{array}\right.
$$

where $\alpha \in(1 / 2,1),{ }_{t} D_{\infty}^{\alpha}$ and ${ }_{-\infty} D_{t}^{\alpha}$ are the right and left inverse operators of the corresponding Liouville-Weyl fractional integrals of order $\alpha$ respectively, $H^{\alpha}$ is the classical fractional Sobolev Space, $u \in \mathbb{R}, b: \mathbb{R} \rightarrow \mathbb{R}, \inf _{t \in \mathbb{R}} b(t)>0$, $f: \mathbb{R} \times \mathbb{R} \rightarrow \mathbb{R}$ Carathéodory function and $M: \mathbb{R}^{+} \rightarrow \mathbb{R}^{+}$is a function that satisfy some suitable conditions.

Fractional differential equations have been receiving great interest recently. This is due to both the intensive development of the theory of fractional calculus itself and the applications of such constructions in various scientific fields such as physics, chemistry, biology, economics, control theory, signal and image processing, biophysics, blood flow phenomena, aerodynamics, fitting of experimental data, etc., [1], [8], [10], [15], [16], [18], [21] and the references therein.

It should be noted that critical point theory and variational methods have also turned out to be very effective tools in determining the existence of solutions for integer order differential equations. The idea behind them is trying to find solutions of a given boundary value problem by looking for critical points of a suitable energy functional defined on an appropriate function space. In the Several years back, the critical point theory has become to a wonderful tool in studying the existence of solutions to differential equations with variational structures, we refer the reader to the books due to Mawhin and Willem [14], Rabinowitz [17] and the references listed therein.

Recently Jiao and Zhou [9], have studied the following fractional boundary value problem

$$
\left\{\begin{array}{l}
{ }_{t} D_{T}^{\alpha}\left({ }_{0} D_{t}^{\alpha} u(t)\right)=\nabla F(t, u(t)), \quad t \in[0, T], \\
u(0)=u(T)=0 .
\end{array}\right.
$$

They proved the existence of solutions to this problem by using critical point theory.

In [20], by using the Mountain Pass Theorem, Torres investigates the existence of solutions for the fractional Hamiltonian systems

$$
\left\{\begin{array}{l}
\left({ }_{t} D_{\infty}^{\alpha}\left({ }_{-\infty} D_{t}^{\alpha} u(t)\right)+L(t) u(t)\right)=\nabla W(t, u(t)), \quad t \in \mathbb{R} \\
u \in H^{\alpha}(\mathbb{R}) .
\end{array}\right.
$$

